\title{
Q uantum dots for tumor-targeted drug delivery and cell imaging
}

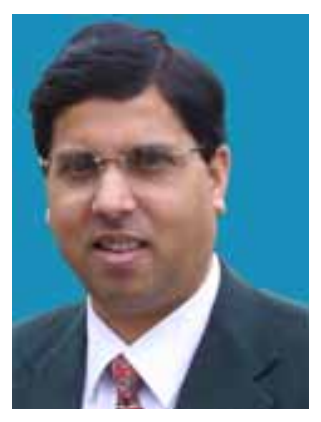

R DK Misra

Center for Structural \& Functional $M$ aterials, University of Louisiana at Lafayette, PO Box 44130, Lafayette, LA 70504-4130, USA

Tel.: +1 337482 6430;

Fax: +1 337482 1220;

E-mail: dmisra@louisiana. edu

\author{
'The drug-loaded chitosan- \\ encapsulated $\mathrm{ZnO}: \mathrm{Mn}^{2+}$ quantum \\ dots represent a potential platform \\ to deliver tumor-ta rgeted drugs \\ and document the delivery \\ process simulta neously.'
}

Early detection of cancer and targeted drug delivery, specifically in and around tumors and at concentrations that would decrease the growth rate and/or viability of the tumors, continues to be the primary challenge in the treatment of cancer. In many cases, the malignancy of tumors is detected only at advanced stages when chemotherapeutic drugs become increasingly toxic to healthy cells. To reduce this problem, targeted drug delivery and early detection of cancer cells continue to be investigated extensively. A tumor-targeting drug delivery system generally consists of a tumor-recognition moiety and a drug-loaded vesicle. Detection systems often involve invasive methods, such as tissue biopsy and sophisticated diagnostics tools, such as magnetic resonance imaging. H owever, there is no known single system currently that is capable of targeting drug delivery and imaging the delivery process simultaneously. An excellent vehicle to accomplish this objective is to develop a single system that is capable of targeting drug delivery and imaging the delivery process simultaneously to monitor the time course of subcellular location. Integration of biomaterials and semiconductor nanocrystal quantum dots (QDS) fields has good potential for addressing the current problems faced in cancer therapy by using biodegradable chitosan ( $\mathrm{N}$-acetyglucosamine) for tumor-targeted drug delivery and Q D s for noninvasive imaging. The drug-loaded chitosan-encapsulated $\mathrm{ZnO}: \mathrm{Mn}^{2+} \mathrm{QD}$ s represent a potential platform to deliver tumor-targeted drugs and document the delivery process simultaneously [H in S, Pers. Comm.].

D oping enables us to tune into desired fluorescence emission. The doping of $\mathrm{ZnO}$ with low concentration of manganese is expected to increase the band-gap energy of $\mathrm{ZnO}$ with a consequent increase in luminescence. C onsidering that the high-molecular-weight chitosan of approximately $200 \mathrm{kD}$ a can increase the size of $Q D$ s, the use of low-molecular-weight chitosan of approximately $50 \mathrm{kD}$ a is preferred. Furthermore, to increase the efficiency of QD solubilization in aqueous medium, fully deacetylated chitosan prepared by thermochemical deacetylation is favored [H in S, Pers. Comm.] [1]. Subsequently, the low-molecular-weight, fully deacetylated chitosan can be conjugated with folic acid for in situ encapsulation of water-dispersible ZnO :M n ${ }^{2+} Q D$ s. A possible flow chart for the synthesis of $\mathrm{Mn}$-doped $\mathrm{ZnO} Q \mathrm{Q}$ s is presented in Figurel, based on some practices reported in the literature $[2,3]$.

The role of diethanolamine (DEA) in Figre1 includes: preventing aggregation of $Q D$ s; coordinating with unsaturated metal atoms on the QD surface to prevent the formation of bulk semiconductors by coordination of $\mathrm{N}$ in DEA with the unsaturated metal ions [2-4]; and providing hydroxide $\left(\mathrm{OH}^{-}\right)$ions for the synthesis and stability of QDS [2]. The surfactant, oleic acid (OA), is believed to have carboxyl groups that react with the hydroxyl group of DEA and forms a complex on the surface of $Q D$ s, resulting in high stability in the aqueous medium [2,3]. Retention of coordinating organic ligands is necessary for maintaining the optical properties of QDs and for shielding the QDs from the environment. The presence of chemically bonded $\mathrm{OA}$ groups on the surface is expected to provide stability to the QDs in the aqueous medium. Furthermore, the hydroxyl group $\left(\mathrm{OH}^{-}\right)$of the DEA molecules will contribute additionally to the stability of nanocrystal QDS in the aqueous environment [2].

Given that a high-molecular-weight chitosan can increase the size of $Q D$ s, use of a low-molecular-weight chitosan of approximately $50 \mathrm{kD}$ a is preferred [Hein S, Pers. Comm.]. Furthermore, to increase the efficiency of $Q D$ solubilization in aqueous medium, fully deacetylated chitosan is favored. Chitosan is a linear polysaccharide copolymer of D-glucosamine (deacetylated unit) and N-acetyl-D-glucosamine (acetylated unit). The degree of deacetylation $(\% D D)$ determines 


\section{Figure 1. Flow chart for the synthesis of water-dispersible Mn-doped ZnO quantum dots.}

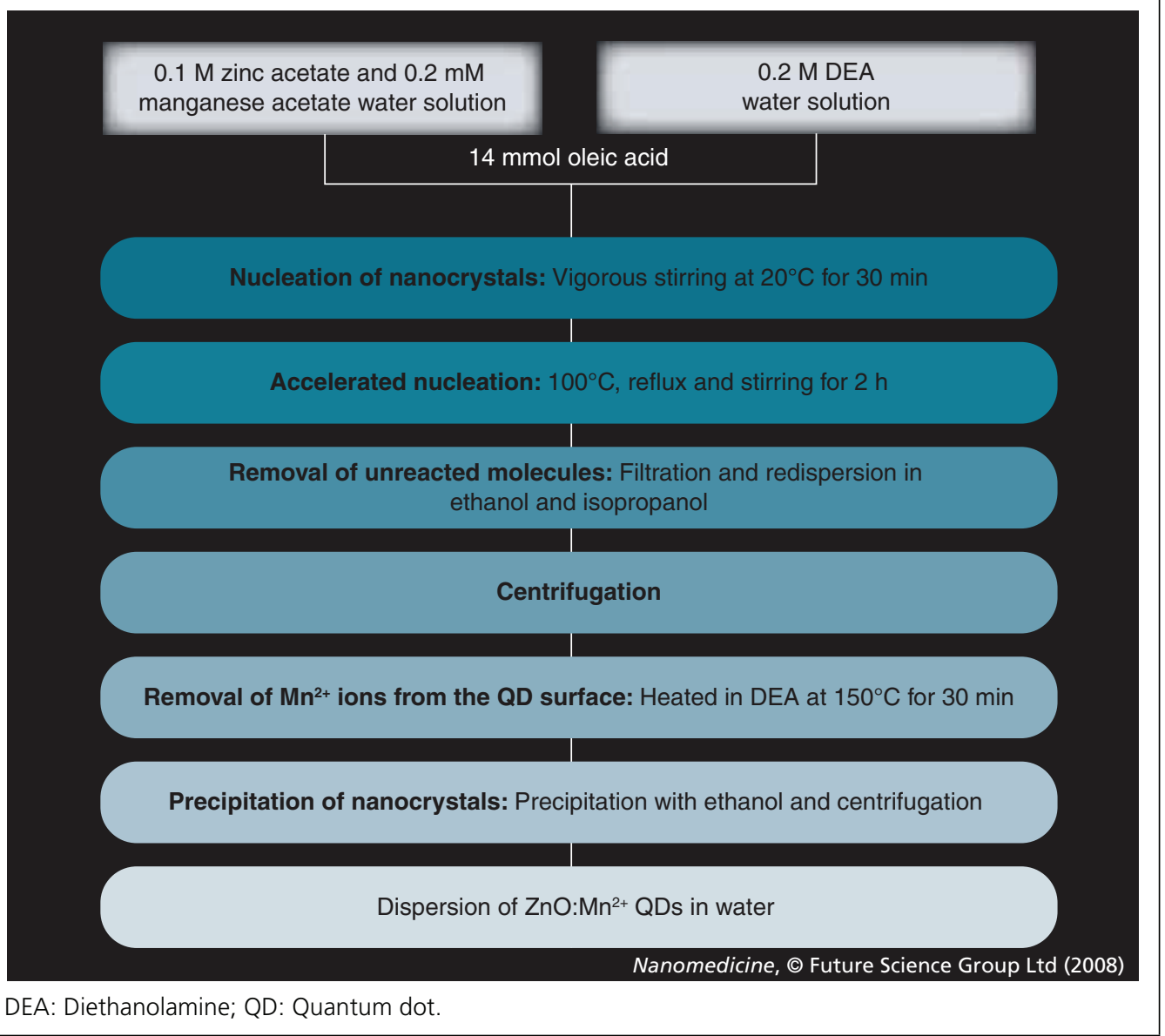

the content of free amino groups in the polysaccharides and is the key chemical characteristic that dictates the physicochemical and biological properties, including sorption property and metal chelation, change of solubility in dilute acid, biocompatibility and biodegradability. $\% \mathrm{DD}$ affects the crystallinity and hydrophilicity of chitosan owing to variations in hydrophilic interactions that ultimately control the ligand attachment, drug loading and release characteristics of chitosan [5-7]. In general, the process of deacetylation involves the removal of an acetyl group $\left(\mathrm{NHCOCH}_{3}\right)$ from the molecular chain and this obtains a complete and chemically reactive amino group $\left(-\mathrm{NH}_{2}\right)$. The functionality and properties of chitosan essentially depend on the chemical-reactive amino group.

Considering that, the preferred approach is to encapsulate $\mathrm{ZnO}: \mathrm{Mn}^{2+}$ QDs with folate-conjugated chitosan. This involves synthesis of the folate-chitosan conjugation, followed by encapsulation of $\mathrm{ZnO}: \mathrm{Mn}^{2+}$ QDs with folate-conjugated chitosan to get folate-chitosan- $\mathrm{ZnO}: \mathrm{Mn}^{2+}$
QD nanoparticles (Figure 2). The nature of a cationic polyelectrolyte that has one amino group and two hydroxyl groups of chitosan provides a strong electrostatic interaction with negative-charged biomolecules and QDs [8]. The folic acid binding covalently to chitosan maintains the nanoparticle size, while keeping a positive-charge ratio of more than +2 . Thus, the low-molecular-weight $(50 \mathrm{kDa})$ and fully deacetylated chitosan can be conjugated with folic acid. Because folic acid is difficult to conjugate to the surface of the polymer, owing to the low reactivity of the carboxylic acid group, the carboxyl group of folic acid is first activated.

It is expected that the drug-loaded, biofunctionalized chitosan-encapsulated QDs will enhance the cellular apoptosis of tumor cells [1]. Recently, it was observed that chitosan exhibits good proliferation of transfected cells [Thein-Han WW, Misra RDK, Unpublished Data]. Moreover, by using drugloaded, biofunctionalized chitosan-encapsulated QDs, the time taken for tumor cells to go into an apoptotic state can also be monitored by 
Figure 2. Representation of the encapsulation of $\mathrm{ZnO}: \mathrm{Mn}^{2+}$ quantum dots with folate-conjugated chitosan (2) and the drug-loading step (3) in which biofunctionalized chitosan loaded with drug is used to coat quantum dots.

Step 1: Preparation of folate-conjugated chitosan

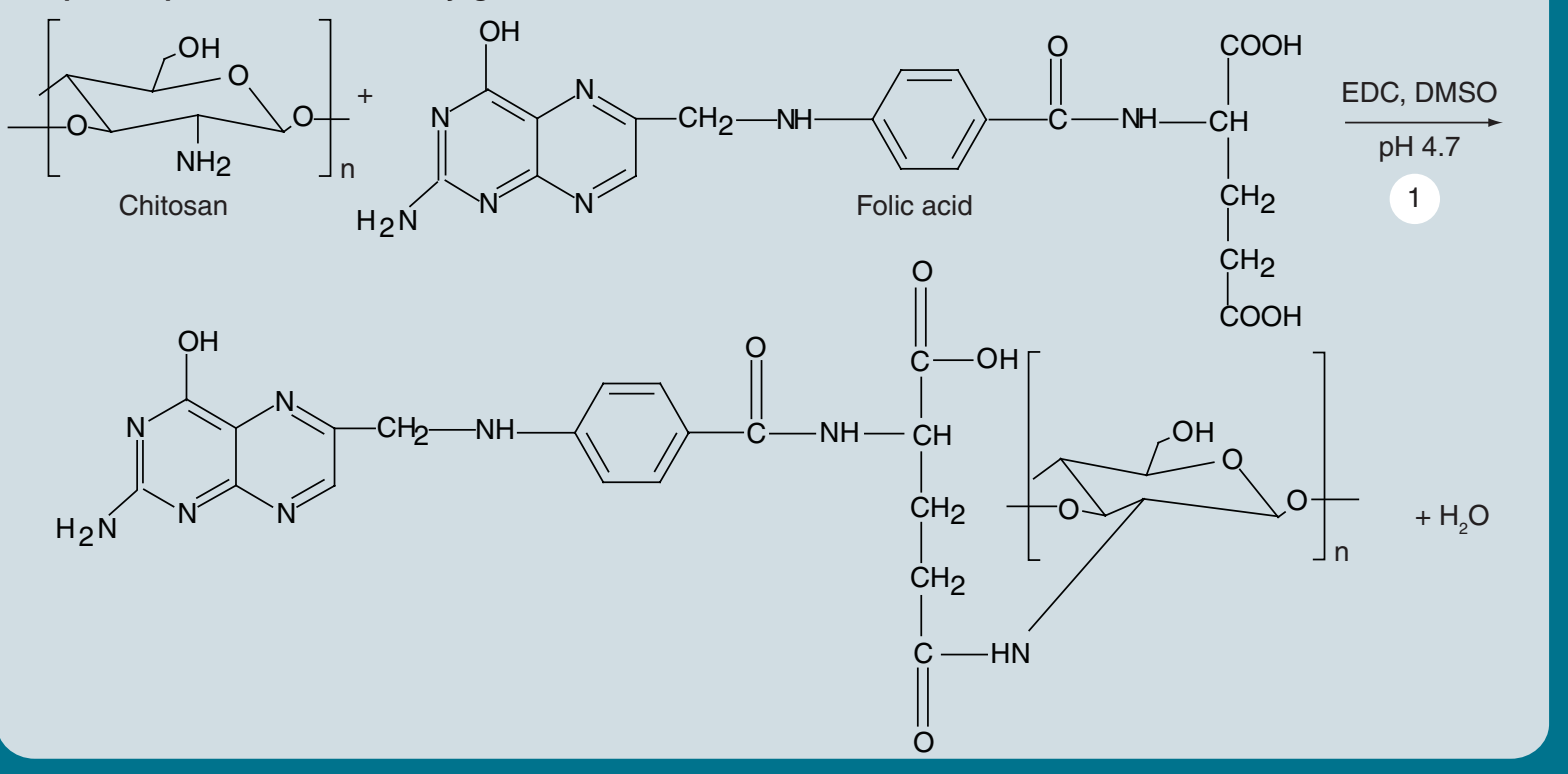

Step 2: Encapsulation of quantum dot with folate-conjugated chitosan

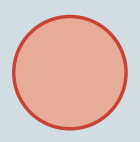

Quantum dot
Folate-conjugated chitosan

(via electrostatic interaction)

2

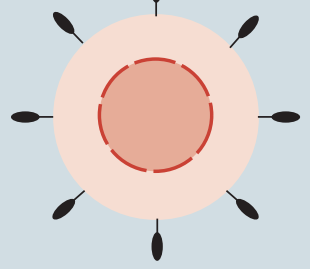

Step 3: Loading with drug

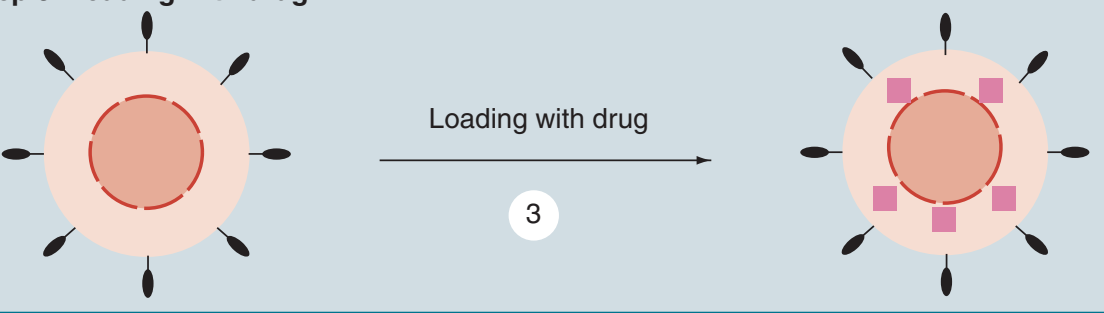

$\mathrm{ZnO}: \mathrm{Mn}^{2+}$ quantum dots

Folic acid

Drug

Electrostatic interaction ,

Nanomedicine, @ Future Science Group Ltd (2008)

confocal laser-scanning microscope. This information will determine the dosage necessary for targeted drug delivery [H in S, Pers. Comm.].

\section{Acknowledgements}

Some of the viewpoints expressed here are an outcome of scientific interaction and communication with San $\mathrm{H}$ ein and those expressed in the open literature. Thus, they are not necessarily solely of the author. Any omission of references is unintentional.
Financial \& competing interests disclosure

The author has no relevant affiliations or financial involve ment with any organization or entity with a financial interest in or financial conflict with the subject matter or materials discussed in the manuscript. This includes employment, consultancies, honoraria, stock ownership or options, expert testimony, grants or patents received or pending, or royalties.

No writing assistance was utilized in the production of this manuscript. 


\section{Bibliography}

1. D omard $A$, Rinado $M$ : Preparation and characterization of fully deacetylated chitosan. Int. J. Biol. M acromol. 5, 49 (1983).

2. Fu YS, Du XW, Sun J, Song SF, Liu J: Single-crystal $\mathrm{ZnO}$ cup based on hydrothermal decomposition route. J. Phys. Chem. C. 111, 3863 (2007).

3. Fu YS, Du XW, Kulinich SA et al.: Stable aqueous dispersion of $\mathrm{ZnO}$ quantum dots with strong blue emission via simple solution route. J. Am. Chem. Soc. 129, 16029-16033 (2007).
4. N orberg N S, Kittilstved KR, Amonette JE, 6. Gupta KC, Jabrail FH : Effects of degree of Kukkadapu RK, Schwartz D A, Gamelin D R: Synthesis of colloidal $\mathrm{M} \mathrm{n}^{2+} \mathrm{ZnO}$ quantum dots and high-TC ferromagnetic nanocrystalline thin films. J. Am. Chem. Soc. 126, 9387-9398 (2004).

5. Kim TH, Park IK, N ah JW, Choi YJ, Cho CS: G alactosylated chitosan/D N A nanoparticles prepared using water-soluble chitosan as a gene carrier. Biomaterials 25, 3783-3792 (2004). deacetylation and cross-linking on physical characteristics, swelling and release behavior of chitosan microspheres. Carbohydr. Polym. 66, 43 (2006)

7. H su SH, W hu SW, Tsai C, Wu YH, Chen $\mathrm{H} \mathrm{W}, \mathrm{H}$ sieh $\mathrm{KH}$ : Chitosan as scaffold materials: effects of molecular weight and degree of deacetylation. J. Polymer Res. 11, 141 (2004).

8. Tan W B, Zhang Y: Surface modification of gold quantum dots nanoparticles. J. Biomed. M ater. Res. A. 75, 56 (2005). 